\title{
Disturbance Observer Based Control of Small Unmanned Aerial Rotorcraft
}

\author{
Xusheng Lei, ${ }^{1,2}$ Kexin Guo, ${ }^{1}$ and Shuzhi Sam Ge ${ }^{2}$ \\ ${ }^{1}$ Science and Technology on Inertial Laboratory, Beijing 100191, China \\ ${ }^{2}$ Department of Electrical Engineering, National University of Singapore, Singapore 119613
}

Correspondence should be addressed to Xusheng Lei; xushenglei@buaa.edu.cn

Received 27 February 2013; Accepted 31 March 2013

Academic Editor: Zheng-Guang Wu

Copyright (c) 2013 Xusheng Lei et al. This is an open access article distributed under the Creative Commons Attribution License, which permits unrestricted use, distribution, and reproduction in any medium, provided the original work is properly cited.

As a complex system, the control performance of small unmanned aerial rotorcraft is easily affected by the dynamic model errors, measurement errors, and environment disturbances. This paper proposes a disturbance observer based control method to improve performance. The disturbance observer based control is constructed by the feedback control and a series of integral filters. The system stability can be guaranteed by the feedback control method. Furthermore, the disturbances can be estimated and eliminated quickly by the integral filters. Therefore, the control performance can be improved effectively. The control performance of the disturbance observer based control has been validated by a series of flight tests. Compared with feedback control, the disturbance observer based control yields a better tracking performance in the presence of disturbances.

\section{Introduction}

With the ability of taking off and landing vertically, as well as hovering, small unmanned aerial rotorcrafts (SUARs) have been widely used in many fields, including city map data acquisition, road traffic monitoring, and power line inspection [1-3].

Since there exist dynamic model errors, measurement errors, and environment disturbances in the flight process of SUAR system, it is hard to realize stable control [4]. Therefore, how to improve the control performance for SUAR system has attracted many attentions in recent years [5]. PID, robust control, and intelligent control methods have been proposed for SUAR system [6-12]. Due to its simple structure, PID control method is often used in SUAR systems $[13,14]$. However, PID control has little robustness to environment disturbances. With high performance in dealing with system disturbances, Robust control, such as $H_{2}, H_{\infty}$, and LQR, are often used to improve system stability $[15,16]$. Robust control has its limitation in system dynamic response characteristics. Intelligent control can cope with parameter uncertainties effectively $[17,18]$. But it needs a lot of training time and sample data to realize stable control [19].
Generally, there exist dynamic model errors, measurement errors, and environment disturbances for SUAR system. The dynamic model errors and measurement errors can be treated as disturbances with certain bound. Disturbance observer based control strategies have been applied in many control areas to improve control performance [20,21]. With the disturbance observer based control, system disturbances can be estimated and compensated effectively. The objective of this paper is to propose a disturbance observer based control method to improve system stability and dynamic response performance. The disturbance observer based control is constructed by the feedback control and a series of integral filters. The system stability can be guaranteed by the feedback control method. Furthermore, the disturbances can be estimated and eliminated quickly by the integral filters. Therefore, the control performance can be improved effectively.

This paper is organized as follows. Section 2 describes the dynamic model of SUAR system. In Section 3, the disturbance observer based control method is presented, and system stability is proved by the Lyapnov function. Then, a series of flight tests validate the effectiveness of the proposed control method in Section 4, followed by the conclusion in Section 5 . 


\section{Dynamic Model of SUAR System}

The dynamic model of SUAR can be derived from a rigid body with six degrees of freedom. The main rotor, tail rotor, horizontal tail, vertical tail, and fuselage generate control forces and moments to realize stable control. Therefore, the dynamic model of SUAR can be defined as follows:

$$
\begin{aligned}
& m \overrightarrow{\dot{V}}+m(\vec{\omega} \times \vec{V})=\vec{F} \\
& I \overrightarrow{\dot{\omega}}+(\vec{\omega} \times I \vec{\omega})=\vec{M}
\end{aligned}
$$

where $m$ is the mass of SUAR system. $\vec{V}=\left[\begin{array}{lll}u & v & w\end{array}\right]^{\prime}$ is velocity vector, and $u, v, w$ are the velocities along the inertial coordinate frame. $\vec{\omega}=\left[\begin{array}{lll}p & q & r\end{array}\right]^{\prime}$ is angular rate vector, and $q, p, r$ are the corresponding angle rates along the body coordinate frame. $\vec{F}=\left[\begin{array}{lll}X & Y & Z\end{array}\right]^{\prime}$ and $\vec{M}=\left[\begin{array}{lll}L & M & N\end{array}\right]^{\prime}$ are vectors of external forces and external moments, respectively. $I$ is the system inertial tensor.

Based on the Newton-Euler equations, the line motion and angular motion of SUAR system can be derived from (1), that is,

$$
\begin{gathered}
\dot{u}=v r-w q-g \sin \theta+\frac{\left(Y_{\mathrm{mr}}+Y_{\mathrm{fus}}+Y_{\mathrm{tr}}+Y_{\mathrm{vf}}\right)}{m}, \\
\dot{v}=w p-u r+g \sin \phi \cos \theta+\frac{\left(X_{\mathrm{mr}}+X_{\mathrm{fus}}\right)}{m}, \\
\dot{w}=u q-v p+g \cos \phi \cos \theta+\frac{\left(Z_{\mathrm{mr}}+Z_{\mathrm{fus}}+Z_{\mathrm{ht}}\right)}{m}, \\
\dot{p}=q r \frac{\left(I_{y y}-I_{z z}\right)}{I_{x x}}+\frac{\left(L_{\mathrm{mr}}+L_{\mathrm{vf}}+L_{\mathrm{tr}}\right)}{I_{x x}}, \\
\dot{q}=p r \frac{\left(I_{z z}-I_{x x}\right)}{I_{y y}}+\frac{\left(M_{\mathrm{mr}}+M_{\mathrm{ht}}\right)}{I_{y y}}, \\
\dot{r}=p q \frac{\left(I_{x x}-I_{y y}\right)}{I_{z z}}+\frac{\left(N_{\mathrm{vf}}+N_{\mathrm{tr}}-Q_{e}\right)}{I_{z z}},
\end{gathered}
$$

where $\theta, \varphi, \psi$ are the pitch, roll, and yaw angle in earth coordinate frame, respectively. The subscript $\mathrm{mr}$, fus, tr, vf, and ht indicate the forces or moments generated by the main rotor, fuselage aerodynamics effects, tail rotor, vertical fin and horizontal tail, respectively. Since weight force acts in the vertical position, it does not produce a moment directly. The corresponding forces generated by the weigh force are introduced by coordinate transformation. $Q_{e}$ is the torque produced by the tail rotor to counteract with the aerodynamic torque on the main rotor blades.

With the relationship between the earth coordinate frame and body coordinate frame, the kinematic transformation matrix of SUAR system can be described by the Euler angles, that is,

$$
\begin{aligned}
{\left[\begin{array}{c}
\dot{\phi} \\
\dot{\theta} \\
\dot{\psi}
\end{array}\right] } & =\left[\begin{array}{ccc}
1 & \tan \theta \sin \phi & \tan \theta \cos \phi \\
0 & \cos \phi & -\sin \phi \\
0 & \frac{\sin \phi}{\cos \theta} & \frac{\cos \phi}{\cos \theta}
\end{array}\right]\left[\begin{array}{l}
p \\
q \\
r
\end{array}\right] \\
= & {\left[\begin{array}{c}
p+\tan \theta(q \sin \phi+r \cos \phi) \\
q \cos \phi-r \sin \phi \\
\frac{(q \sin \phi+r \cos \phi)}{\cos \theta}
\end{array}\right] . }
\end{aligned}
$$

For SUAR system, Bell-Hiller stabilizer bar is used as a lagged rate feedback to improve system stability. Since the flapping motion is a periodic motion, the general solution for the flapping equation can be represented by an Infinite Fourier Series. Therefore, the dynamic model of rotor flapping motion can be defined as follows:

$$
\begin{gathered}
\dot{a}_{1 p}=-q+K_{b} p-K_{a} a_{1 p}+K_{2} B_{1 s}, \\
\dot{b}_{1 p}=K_{b} q-p-K_{a} b_{1 p}+K_{1} A_{1 s},
\end{gathered}
$$

where $a_{1 p}$ and $b_{1 p}$ are longitudinal blade angle and lateral blade angle, respectively. $A_{1 s}$ and $B_{1 s}$ are longitudinal cyclic input and lateral cyclic input, respectively. $K_{b}, K_{a}, K_{1}, K_{2}$ are identification parameters.

With the small perturbation theory, the dynamic equation and kinematic equation of SUAR can be linearized to reduce computation complexity. Therefore, the dynamic model of SUAR can be constructed as follows:

$$
\dot{x}(t)=A x(t)+B(u(t)+d(t)),
$$

where $x=\left[\theta, \phi, \psi, u, v, w, q, p, r, a_{1 p}, b_{1 p}\right]^{T} \in R^{n}$ is state variable, representing the corresponding angle, velocity, angle velocity, and blade angle information. $u \in\left[A_{1 s}, B_{1 s}, A_{M}\right.$, $\left.A_{T}\right]^{T} \in R^{m}$ is control input, representing longitudinal cyclic input, lateral cyclic input, collective and heading, respectively. $n$ and $m$ are the dimension of state variable and control input, respectively. $A \in R^{n \times n}$ and $B \in R^{n \times m}$ are the coefficient matrixes. $d \in R^{m}$ is the bounded disturbance, denoting wind disturbances, atmospheric turbulences, measurement errors, and system parametric uncertainties of SUAR system.

Assumption 1. The $(A, B)$ is controllable.

Assumption 2. The disturbance $d(t)$ is bounded with an unknown bound.

The control objective is to construct the control strategy to estimate and eliminate the disturbances effectively.

\section{Disturbance Observer Based Control}

From Assumption 1, the SUAR system is controllable. Thus, the control input can be constructed by two parts. One is the state feedback control input $K x(t)$, and the other is the estimation of system disturbances $\widehat{\omega}(t)$, that is,

$$
u(t)=-\widehat{\omega}(t)+K x(t),
$$


where $\widehat{\omega}(t)$ is the estimation value of system disturbances, and $K$ is the weight matrix of the feedback control.

Therefore, the dynamic model of the SUAR system can be transformed as follows:

$$
\dot{x}(t)=(A+B K) x(t)+B(-\widehat{\omega}(t)+d(t)) .
$$

Define the error between the desired state variable $x_{d}$ and the real state variable $x$ as

$$
e=x-x_{d} .
$$

Therefore, the error dynamic model can be defined as follows:

$$
\dot{e}(t)=(A+B K) e(t)+B(-\widehat{\omega}(t)+\omega(t)),
$$

where $B \omega(t)=B d(t)+(A+B K) x_{d}-\dot{x}_{d}$.

Since $x_{d}(t)$ is the state variable with upper bound, $\dot{x}_{d}(t)$ is the state variable with upper bound. Based on Assumption 2, $d(t)$ is the system disturbance with bound. Thus, $\omega(t)$ is a function vector with bound. If the disturbance $\omega(t)$ can be estimated by the disturbance observer $\widehat{\omega}(t)$, the control performance can be improved effectively.

Lemma 1. Considering the following integral filters:

$$
\begin{aligned}
& \dot{\eta}(t)=-\alpha \eta(t)+(\alpha-\beta) e^{-\beta t} \eta(0)+\int_{0}^{t} e^{-\alpha(t-s)} B \omega(s) d s, \\
& \dot{\hat{\eta}}(t)=-\alpha \widehat{\eta}(t)+(\alpha-\beta) e^{-\beta t} \widehat{\eta}(0)+\int_{0}^{t} e^{-\alpha(t-s)} B \widehat{\omega}(s) d s,
\end{aligned}
$$

where $\alpha$ and $\beta$ are positive constant values. $\eta(0)$ and $\hat{\eta}(0)$ are initial values, and $\widehat{\omega}(t)$ is the estimation value of $\omega(t)$.

Define the error between the estimation value $\widehat{\omega}(t)$ and real value $\omega(t)$ as

$$
\widetilde{\omega}(t)=\widehat{\omega}(t)-\omega(t) .
$$

With the integral filters, the true value $\omega(t)$ can be converged by the estimation value $\widehat{\omega}(t)$ quickly, that is,

$$
|B \widetilde{\omega}(t)| \leq \delta e^{-\sigma t},
$$

where $\delta, \sigma$ are positive constant values.

The estimation value $\widehat{\omega}(t)$ can be obtained from the following integral equation:

$$
\int_{0}^{t} e^{\alpha s} B \widehat{\omega}(s) d s=\alpha e^{\alpha s} \int_{0}^{t} e^{-\alpha s} \psi(s) d s+\psi(t),
$$

where

$$
\begin{aligned}
\psi(t)= & (\beta-\gamma) e^{(\alpha+\gamma-\beta) t}(\widehat{\eta}(0)-\eta(0)) \\
& +\beta e^{(\alpha-\beta) t} \eta(0)+\left(e^{\gamma t}-1\right) \\
& \times \int_{0}^{t} e^{\alpha s} v(s) d s+\left(\alpha+(\gamma-\alpha) e^{\gamma t}\right) \\
& \times \int_{0}^{t}\left[\theta(s)+\int_{0}^{s} e^{\alpha s} v(r) d r\right] d s \\
& +\left(r^{\gamma t}-1\right) \theta(t),
\end{aligned}
$$

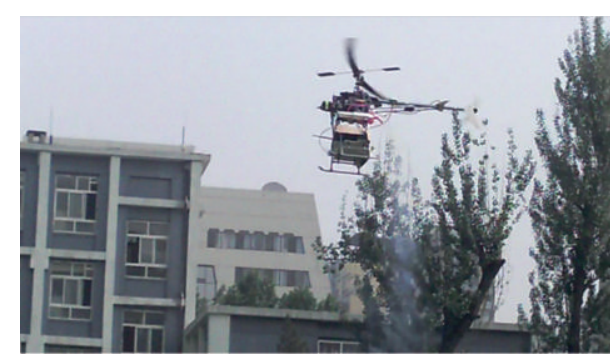

FIGURE 1: The SUAR system.

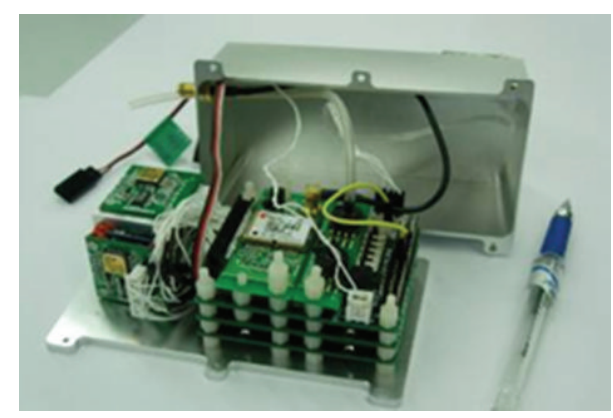

Figure 2: The MGNC system.

where $v(t)=-(A+B K) e(t), \theta(t)=e^{\alpha t} e(t)-e(0)-$ $\alpha \int_{0}^{t} e^{\alpha s} e(s) d s$.

The proof can be found in Appendix A.

Theorem 2. According to Lemma 1, with the controller (6) and the observer (10), the system (5) is stable. The system disturbances can be estimated and eliminated effectively, and the

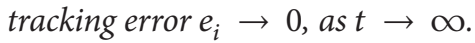

The proof can be found in Appendix B.

\section{Flight Test}

The SUAR system is constructed by a radio-controlled hobby helicopter, shown in Figure 1. The SUAR is $1.46 \mathrm{~m}$ in span and $1.3 \mathrm{~m}$ in length. The total weight is $5 \mathrm{~kg}$. The SUAR is powered by a piston engine running on a mixture of methanol and oil. Five motors are used to control throttle, longitudinal cyclic input, lateral cyclic input, collective and heading. With the constraint of weight and size, the micro guidance, navigation and control (MGNC) system with small weight is developed to realize stable control, shown in Figure 2. The MGNC is only $207 \mathrm{~g}$. It consists of a horizontal main board, housing three angular rate gyros and two 2-axis accelerometers. The angular rate gyro employs the LCG50 gyro that the constant drift is 80 degree/hour. The accelerometer employs the Model 1221 that the bias is $1 \mathrm{mg}$. The Novatel RTK DGPS is utilized to provide position and velocity information. And the bias error is $0.3 \mathrm{~m}$. Furthermore, the compass that was generated by the Honeywell is used to provide heading information and the measurement error is less than 1 degree. Moreover, Motorola MPXA 6115 is chosen as the barometer to provide 
altitude information. Based on the ARM chip AT91RM200 and a DSP chip ADSP21364, MGNC system can get sensor information from angular rate gyros, accelerometers, DGPS, and barometer sensors. With the adaptive KF algorithm, the attitude error in pitch and roll angles are less than 0.5 degree, and the error for yaw angle is less than 0.8 degree. The navigation frequency is $50 \mathrm{~Hz}$ [20].

The flight control system of SUAR is composed by the flight scheduling layer and the kernel control layer. The flight scheduling layer is to guarantee the trajectory following performance for the preschedule flight tasks. Based on the DGPS and compass information, system generates the planned attitude angles to keep it asymptotically stable. The kernel control layer is to realize attitude control. Based on the proposed method, the system adjusts throttle, longitudinal cyclic input, lateral cyclic input, collective and heading to realize high performance control. The update cycles of the kernel control layer and the flight scheduling layer are $20 \mathrm{~ms}$ and $100 \mathrm{~ms}$, respectively.

With the adaptive GA, the weight matrixes $A \in R^{n \times n}$ and $B \in R^{n \times m}$ can be got from the flight data [21]:

$$
\begin{aligned}
& A=\left[\begin{array}{cccccccccccc}
0 & 0 & 0 & 0 & 0 & 0 & 0 & 1 & 0 & 0 & 0 & 0 \\
0 & 0 & 0 & 0 & 0 & 0 & 0 & 0 & 1 & 0 & 0 & 0 \\
0 & 0 & 0 & 0 & 0 & 0 & 0 & 0 & 0 & 1 & 0 & 0 \\
1.2481 & 0 & 0 & 0.9934 & 0 & 0 & 0 & 0 & 0 & 0 & 0 & 0 \\
0 & 0.8147 & 0 & 0 & 0 & 1.0002 & 0 & 0 & 0 & 0 & 0 & 0 \\
0 & 0 & 0 & 0 & 0 & 0 & 1.0013 & 0 & 0 & 0 & 0 & 0 \\
0 & 0 & 0 & -0.2347 & 0 & -0.0114 & 0 & -0.4402 & 0 & 0 & 8.743 & 4.261 \\
0 & 0 & 0 & -0.0738 & 0 & -0.0415 & 0 & 0 & -0.4396 & 0 & -3.412 & 5.743 \\
0 & 0 & 0 & 0 & 0 & 0 & 0.0001 & 0 & 0 & -0.0245 & 0 & 0 \\
0 & 0 & 0 & 0 & 0 & 0 & 0 & -1 & 0.0647 & 0 & -11.456 & 0 \\
0 & 0 & 0 & 0 & 0 & 0 & 0 & 0.0647 & -1 & 0 & 0 & -11.456
\end{array}\right], \\
& B=\left[\begin{array}{cccc}
0 & 0 & 0 & 0 \\
0 & 0 & 0 & 0 \\
0 & 0 & 0 & 0 \\
0 & -1.1386 & 0 & 0 \\
-0.7954 & 0 & 0 & 0 \\
0 & 0 & -0.1284 & 0 \\
0.4279 & 1.7823 & 0 & 0 \\
-0.3112 & -1.1356 & 0 & 0 \\
0 & 0 & 0 & -1.1385 \\
0 & 15.8763 & 0 & 0 \\
15.8763 & 0 & 0 & 0
\end{array}\right] .
\end{aligned}
$$

Based on the pole assignment theory, the feedback control matrix $K$ can be defined as

$$
K=\left[\begin{array}{ccccccccccc}
4.3580 & 2.0151 & -0.6039 & 3.8038 & 2.8587 & 0.3234 & 1.4260 & 1.7467 & 0.3440 & 0.3215 & 0.1148 \\
8.2037 & -2.7753 & -1.4599 & 7.7734 & -3.2731 & 0.1765 & 1.6774 & -1.4569 & 0.3853 & 0.4916 & -0.2029 \\
-1.1356 & 0.1203 & -0.8201 & 0.3201 & 0.0003 & -13.1023 & -4.0077 & 5.6982 & -1.4049 & -4.1813 & 7.2079 \\
31.6250 & -57.4912 & -76.9994 & 29.5218 & -67.0971 & 0.2203 & 34.2618 & -19.6663 & -7.3486 & 24.9242 & -0.8321
\end{array}\right] .
$$

To test the effectiveness of the disturbance observer based control method, a comparison flight test between the feedback control and the disturbance observer based control has been done on the SUAR system. The same feedback control parameter $K$ was chosen for the feedback control method and the disturbance observer based control method. The flight task included a straight flight with low speed and a hovering flight test. The start point was set as $(0,-100,20)$, and the end point was set as $(0,0,20)$. The SUAR flied from the start point to end point directly and then hovered at the end point. The largest and the smallest wind disturbances were $4.37 \mathrm{~m} / \mathrm{s}$ and $2.41 \mathrm{~m} / \mathrm{s}$, respectively. The flight trajectories generated by the feedback control method and the disturbance observer based control are shown in Figure 3. The corresponding pitch and roll angles are shown in Figures 4 and 5, respectively. The dot line and solid line are the control trajectories generated by the feedback control and the disturbance observer based control, respectively. From Figures $3-5$, it is easy to see that the two control methods can realize stable control under wind disturbances. In the straight flight with low speed phase, 


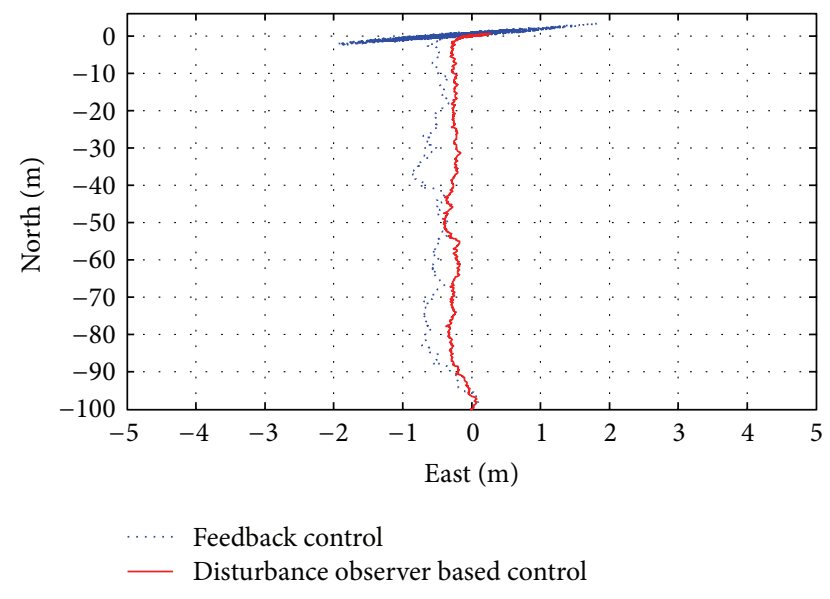

FIGURE 3: The comparison of flight trajectories between the feedback control and disturbance observer based control under wind disturbance.

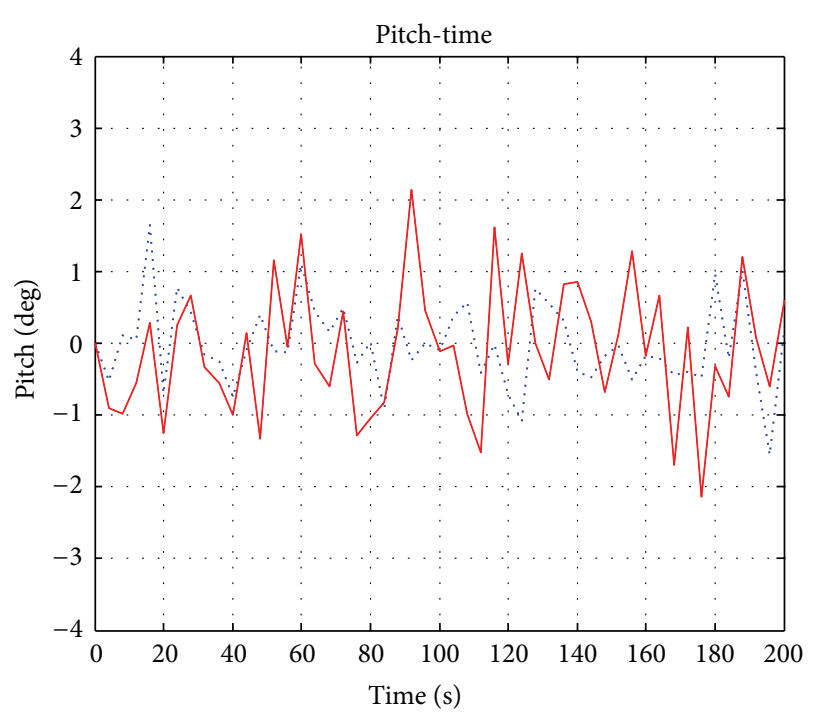

Feedback control

— Disturbance observer based control

Figure 4: The comparison of pitch angle between the feedback control and disturbance observer based control under wind disturbance.

the maximum deviation from the planned trajectories and the mean deviation error generated by the disturbance observer based control are $0.37 \mathrm{~m}$ and $0.18 \mathrm{~m}$, respectively, that are nearly 38 percent of the values generated by the feedback control method. For the feedback control method, there exists a large distance deviation from the end point due to wind disturbance, and the maximum deviation is $1.97 \mathrm{~m}$. The disturbance observer based control can make a good estimation for wind disturbances, and the maximum distance deviation from the end point is $0.35 \mathrm{~m}$. The largest pitch and roll angles are 2.1 degree and 4.2 degree, respectively, that are the permission range of SUAR system.

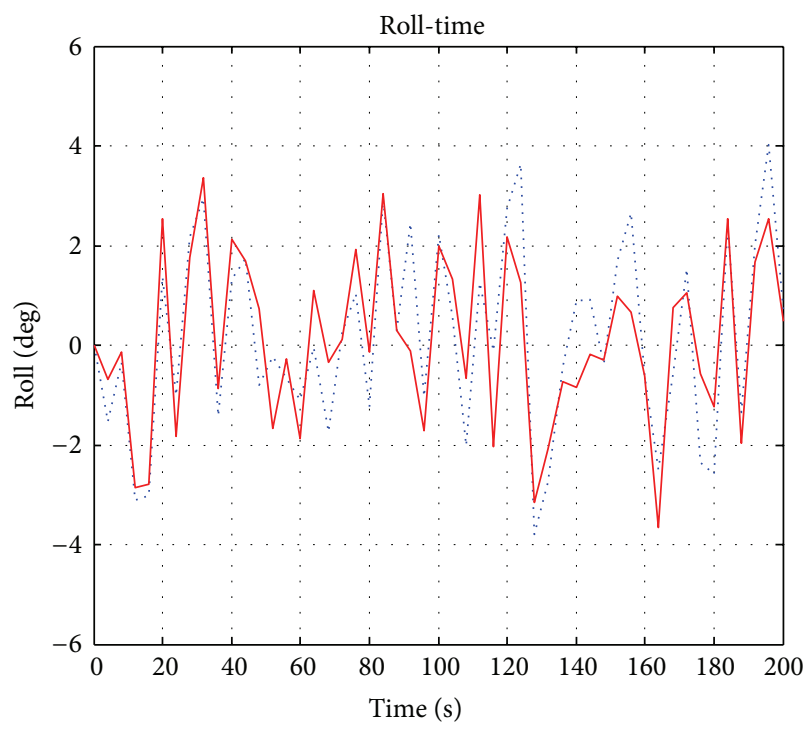

Feedback control

_ Disturbance observer based control

FIGURE 5: The comparison of roll angle between the feedback control and disturbance observer based control under wind disturbance.

Furthermore, a rectangle cruise flight with different height has also been tested on the SUAR system. Point $1(0$, $0,20)$, point $2(50,0,20)$, point $3(50,30,20)$, point $4(50,30$, $30)$, point $5(50,50,30)$, point $6(0,50,30)$, point $7(0,30,30)$, and point $8(0,30,20)$ were set as the hovering points. When the SUAR received the auto pilot command, it hovered at the point 1 , and then adjusted the heading based on the coordinate relationship between point 1 and point 2 . When the error of position and speed satisfied the criteria threshold of hovering stage, the SUAR flied to point 2 . With the same flight process, the SUAR cruised hovering point 1-8 and then hovered at the point 1 . In the flight process, the SUAR pulled up the height from point 3 to point 4 , and dropped the height form point 7 to point 8 . The wind disturbance was from $2.3 \mathrm{~m} / \mathrm{s}$ to $3.8 \mathrm{~m} / \mathrm{s}$, measured by the anemometer. The 3D flight trajectories and the projection of 3D flight trajectories on the coordinate plane of the east and north are shown in Figures 6 and 7, respectively. The dot line, solid line, and dash line are the planned trajectories, the control trajectories generated by the disturbance observer based control and the feedback control, respectively. The corresponding pitch and roll angles are shown in Figures 8 and 9, respectively. For the disturbance observer based control, the maximum deviation error is $1.21 \mathrm{~m}$ that is similar with value generated by the feedback control method, but the mean error is $0.47 \mathrm{~m}$ that is nearly 68 percent of the mean error generated by the feedback control method. In the flight process, the disturbance observer based control could make a good estimation for system disturbances and made the corresponding adjustments to improve control performance. The ranges of pitch angle and roll angle are 9.3 degree and 3.1 degree, respectively, that is less than the values generated by the feedback control method. 


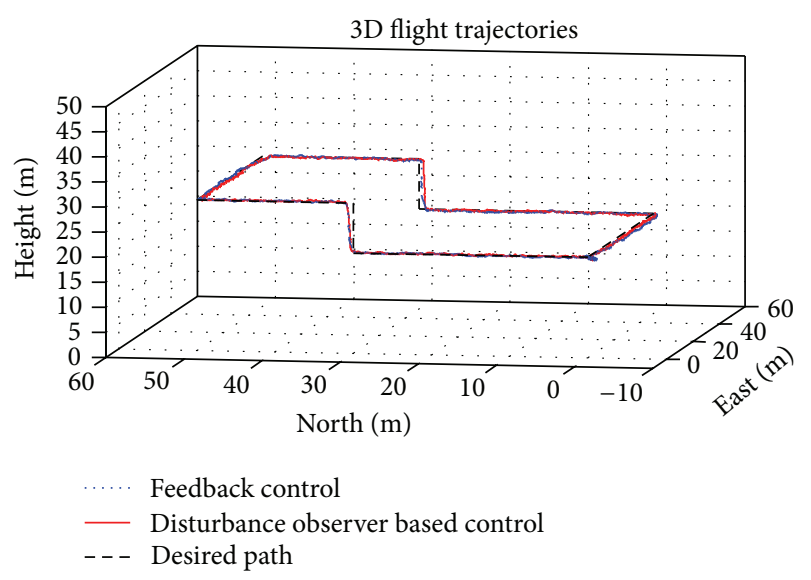

FIGURE 6: The comparison of flight trajectories of the SUAR using the feedback control and the disturbance observer based control.

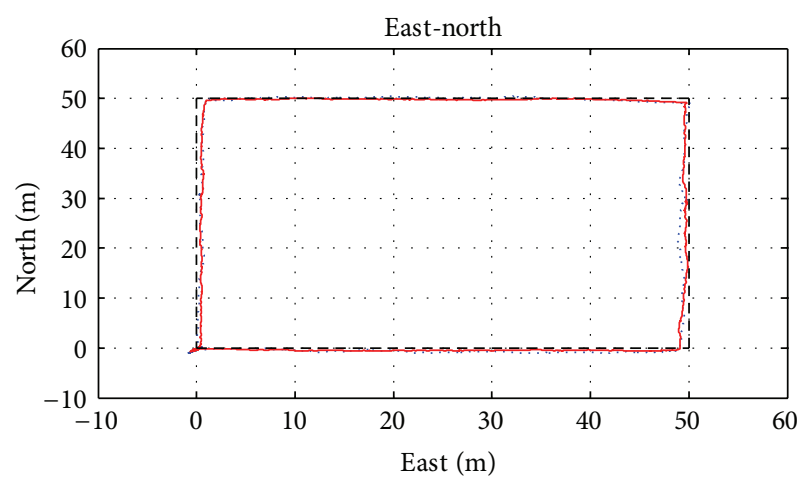

Feedback control

_ Disturbance observer based control

- - Desired path

Figure 7: The projection of 3D flight trajectories on the coordinate plane of east and north.

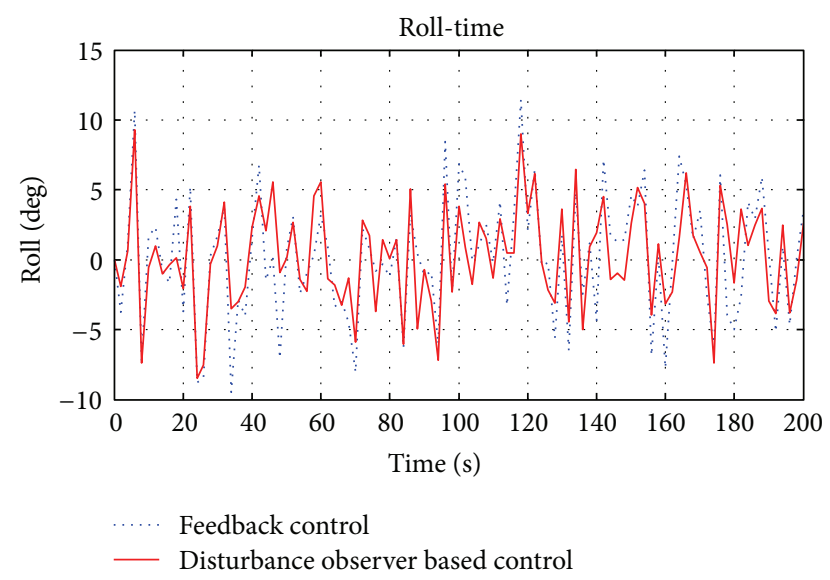

FIgURE 8: The roll angles of the SUAR using the feedback control and the disturbance observer based control.

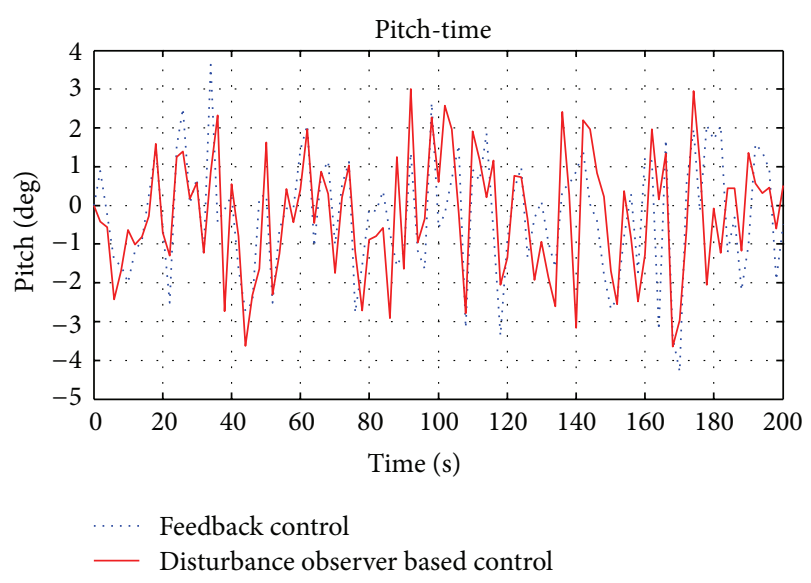

FIGURE 9: The pitch angles of the SUAR using the feedback control and the disturbance observer based control.

\section{Conclusion}

In this paper, a disturbance observer based control method is proposed to deal with system disturbances in flight process. Based on the feedback control, system stability can be guaranteed. With a series of integral filters, the disturbances can be estimated and eliminated quickly, thus the control performance can be improved effectively. The effectiveness of the disturbance observer based control has been demonstrated by a series of hovering and straight flight tests for the SUAR system. The maximum deviation from the planned trajectories and the mean position error generated are $0.37 \mathrm{~m}$ and $0.18 \mathrm{~m}$, respectively, in the straight flight process, that are nearly 38 percent of the values generated by the feedback control method. Since the same feedback control parameters are used for the disturbance observer based control method and feedback control method, the flight tests show that the disturbance observer based control can make a good estimation for system disturbances and improve the control performance effectively.

\section{Appendices}

\section{A. Proof of Lemma 1}

Consider the following differential equation:

$$
\dot{\hat{\eta}}(t)-\dot{\eta}(t)+\gamma(\widehat{\eta}(t)-\eta(t))+e^{-\gamma t} \dot{\eta}(t)=0 .
$$

Its solution is $\widehat{\eta}(t)=\left(1-e^{-\gamma t}\right) \eta(t)+e^{-\gamma t} \eta(0)$.

Thus, $\widehat{\eta}(t)$ can converge to $\eta(t)$ quickly.

For (10), the solution is

$$
\begin{aligned}
\widehat{\eta}(t)= & e^{-\alpha t} \widehat{\eta}(0) \\
& +e^{-\alpha t} \int_{0}^{t} e^{\alpha s}\left[(\alpha-\beta) e^{-\beta s} \widehat{\eta}(0)+\int_{0}^{s} e^{-\alpha(s-r)} B \widehat{\omega}(r) d r\right] d s,
\end{aligned}
$$


$\eta(t)=e^{-\alpha t} \eta(0)$

$$
+e^{-\alpha t} \int_{0}^{t} e^{\alpha s}\left[(\alpha-\beta) e^{-\beta s} \eta(0)+\int_{0}^{s} e^{-\alpha(s-r)} B \omega(r) d r\right] d s .
$$

Submit (A.2) into (A.1), then

$$
\int_{0}^{t} e^{\alpha s} B \widetilde{\omega}(s) d s+(\gamma-\alpha) \int_{0}^{t} \int_{0}^{s} e^{-r s} B \widetilde{\omega}(r) d r d s=\phi(t),
$$

where

$$
\begin{aligned}
\phi(t)= & (\beta-\gamma) e^{(\alpha-\beta) t}(\widehat{\eta}(0)-\eta(0)) \\
& +\beta e^{(\alpha-\beta-\gamma) t} \eta(0)-e^{-\gamma t} \int_{0}^{t} e^{\alpha s} B \omega(s) d s \\
& +\alpha e^{-\gamma t} \int_{0}^{t} \int_{0}^{s} e^{\alpha r} B \omega(r) d r d s .
\end{aligned}
$$

Defining the auxiliary variable

$$
\Phi(t)=\int_{0}^{t} \int_{0}^{s} e^{\alpha r} B \widetilde{\omega}(r) d r d s
$$

Differentiating (A.5), then

$$
\dot{\Phi}(t)=\int_{0}^{s} e^{\alpha r} B \widetilde{\omega}(r) d r .
$$

Submitting (A.5) and (A.6) into (A.3)

$$
\dot{\Phi}(t)=-(\gamma-\alpha) \Phi(t)+\phi(t) .
$$

The solution is

$\Phi(t)=e^{-(\gamma-\alpha) t} \int_{0}^{t} e^{(\gamma-\alpha) s} \phi(s) d s+e^{-(\gamma-\alpha) t} \phi(0)$.

\section{Differentiating (A.7)}

$$
\begin{aligned}
\ddot{\Phi}(t)= & -(\gamma-\alpha)^{2} e^{-(\gamma-\alpha) t} \int_{0}^{t} e^{(\gamma-\alpha) s} \phi(s) d s \\
& -(\gamma-\alpha) \phi(t)+\dot{\phi}(t) .
\end{aligned}
$$

Differentiating (A.6), then

$$
\ddot{\Phi}(t)=e^{\alpha t} B \widetilde{\omega}(t)
$$

Submitting (A.10) to (A.9)

$$
\begin{aligned}
B \widetilde{\omega}(t)= & -(\gamma-\alpha)^{2} e^{-\gamma t} \int_{0}^{t} e^{(\gamma-\alpha) s} \phi(s) d s \\
& -e^{-\alpha t}(\gamma-\alpha) \phi(t)+e^{-\alpha t} \dot{\phi}(t) .
\end{aligned}
$$

Submitting (A.3) to (A.11)

$$
B \widetilde{\omega}(t)=\left[\begin{array}{l}
c_{11} \\
c_{21} \\
c_{31} \\
c_{41} \\
c_{51} \\
c_{61} \\
c_{71} \\
c_{81}
\end{array}\right] e^{-\beta t}+\left[\begin{array}{l}
c_{12} \\
c_{22} \\
c_{32} \\
c_{42} \\
c_{52} \\
c_{62} \\
c_{72} \\
c_{82}
\end{array}\right] e^{-\gamma t}+\left[\begin{array}{l}
c_{13} \\
c_{23} \\
c_{33} \\
c_{43} \\
c_{53} \\
c_{63} \\
c_{73} \\
c_{83}
\end{array}\right] e^{-(\beta+\gamma) t}
$$

where $c_{i 1}, c_{i 2}$, and $c_{i 3}$ are constant values, $i=1,2, \ldots, 8$. Therefore,

$$
|B \widetilde{\omega}(t)| \leq \delta e^{-\sigma t},
$$

where $\sigma=\min (\beta, \gamma)$.

From (7)

$$
B \omega(t)=\dot{e}(t)+B \widehat{\omega}(t)-(A+B K) e(t) .
$$

Submitting the (A.14) to the (A.3)

$$
\int_{0}^{t} e^{\alpha s} \widehat{\omega}(s) d s-\alpha \int_{0}^{t} \int_{0}^{s} e^{-\alpha s} \widehat{\omega}(r) d r d s=\psi(t),
$$

where

$$
\begin{aligned}
& \psi(t)=(\beta-\gamma) e^{(\alpha-\beta-\gamma) t}(\widehat{\eta}(0)-\eta(0)) \\
&+\beta e^{(\alpha-\beta) t} \eta(0)+\left(e^{\gamma t}-1\right) \int_{0}^{t} e^{\alpha s} v(s) d s \\
&+\left[\alpha+(\gamma-\alpha) e^{\gamma t}\right] \int_{0}^{t}\left[\theta(s)+\int_{0}^{s} e^{\alpha r} v(r) d r\right] d s \\
&+\left(e^{r t}-1\right) \theta(t), \\
& \theta(t)=e^{\alpha t} e(t)-e(0)-\int_{0}^{t} e^{\alpha s} e(s) d s, \\
& v(t)=-(A+B K) e(t) .
\end{aligned}
$$

Based on (A.5), the following equation can be got:

$$
\int_{0}^{t} e^{\alpha s} B \widehat{\omega}(s) d s=\alpha e^{\alpha s} \int_{0}^{t} e^{-\alpha s} \psi(s) d s+\psi(t),
$$

where

$$
\begin{aligned}
\psi(t)= & (\beta-\gamma) e^{(\alpha+\gamma-\beta) t}(\widehat{\eta}(0)-\eta(0)) \\
& +\beta e^{(\alpha-\beta) t} \eta(0)+\left(e^{\gamma t}-1\right) \int_{0}^{t} e^{\alpha s} v(s) d s \\
& +\left(\alpha+(\gamma-\alpha) e^{\gamma t}\right) \int_{0}^{t}\left[\theta(s)+\int_{0}^{s} e^{\alpha s} v(r) d r\right] d s \\
& +\left(r^{\gamma t}-1\right) \theta(t) .
\end{aligned}
$$

The proof is completed. 


\section{B. Proof of Theorem 2}

According to Lemma 1, the error between the estimate value and the real disturbance is

$$
|B \widetilde{\omega}(t)| \leq \delta e^{-\sigma t}
$$

Hence, with the $t \rightarrow \infty$, the

$$
|B \widetilde{\omega}(t)| \longrightarrow 0 \text {. }
$$

The disturbance estimation $\widehat{\omega}(t)$ can converge to its true value $\omega(t)$.

For error dynamic model of SUAR in (4), consider the nonnegative function

$$
V=\frac{1}{2} e^{T} P e
$$

where the $P$ is the symmetric definite matrix solution to

$$
(A+B K)^{T} P+P(A+B K)=-Q
$$

where $Q$ is the symmetric definite matrix solution.

Thus, the time derivative of the nonnegative function can be defined as follows

$$
\dot{V}=e^{T} \dot{e}=-e^{T} Q e+2 e^{T} B(\omega(t)-\widehat{\omega}(t)) .
$$
holds:

By using Young's inequality, the following inequality

$$
\begin{aligned}
e^{T} B(\omega(t)-\widehat{\omega}(t)) & \leq\left|e^{T} B(\omega(t)-\widehat{\omega}(t))\right| \\
& \leq\left|e^{T}\right||B(\omega(t)-\widehat{\omega}(t))| \leq\left|e^{T}\right| \delta e^{-\sigma t}
\end{aligned}
$$

Therefore,

$$
\dot{V} \leq-e^{T} Q e+\left|e^{T}\right| \delta e^{-\sigma t} .
$$

With the $t \rightarrow \infty$, the

$$
\delta e^{-\sigma t} \longrightarrow 0
$$

Then, $\dot{V} \leq 0$, as $t \rightarrow \infty$.

That is, the error dynamic model of the SUAR system is asymptotically stable.

Then, it can prove that the tracking error $e \rightarrow 0$, as $t \rightarrow$ $\infty$.

The proof is completed.

\section{Acknowledgments}

The research is supported by the National Natural Science Foundation of China (Grant no. 61273033, 60905056, and 60904093), and Beijing Municipal Natural Science Foundation (Grant no. 4132043).

\section{References}

[1] A. J. de Voogt, S. Uitdewilligen, and N. Eremenko, "Safety in high-risk helicopter operations: the role of additional crew in accident prevention," Safety Science, vol. 47, no. 5, pp. 717-721, 2009.

[2] I. Maza, K. Kondak, M. Bernard, and A. Ollero, "Multi-UAV cooperation and control for load transportation and deployment," Journal of Intelligent and Robotic Systems, vol. 57, no. 1-4, pp. 417-449, 2010.

[3] N. Metni and T. Hamel, "A UAV for bridge inspection: visual servoing control law with orientation limits," Automation in Construction, vol. 17, no. 1, pp. 3-10, 2007.

[4] G. W. Cai, B. M. Chen, T. H. Lee, and M. B. Dong, "Design and implementation of a hardware-in-the-loop simulation system for small-scale UAV helicopters," Mechatronics, vol. 19, no. 7, pp. 1057-1066, 2009.

[5] P. Fabiani, V. Fuertes, A. Piquereau, R. Mampey, and F. TeichteilKönigsbuch, "Autonomous flight and navigation of VTOL UAVs: from autonomy demonstrations to out-of-sight flights," Aerospace Science and Technology, vol. 11, no. 2-3, pp. 183-193, 2007.

[6] C. J. Liu, W. H. Chen, and J. Andrews, "Tracking control of small scale helicopters using explicit nonlinear MPC augmented with disturbance observers," Control Engineering Practice, vol. 20, pp. 258-268, 2012.

[7] J. Gadewadikar, F. L. Lewis, K. Subbarao, K. Peng, and B. M. Chen, "H-infinity static output-feedback control for rotorcraft," Journal of Intelligent and Robotic Systems, vol. 54, no. 4, pp. 629646, 2009.

[8] K. M. Peng, G. W. Cai, B. M. Chen, M. B. Dong, K. Y. Lum, and T. H. Lee, "Design and implementation of an autonomous flight control law for a UAV helicopter," Automatica, vol. 45, no. 10, pp. 2333-2338, 2009.

[9] L. Marconi and R. Naldi, "Robust full degree-of-freedom tracking control of a helicopter," Automatica, vol. 43, no. 11, pp. 1909-1920, 2007.

[10] C. H. Luo, R. F. Liu, C. D. Yang, and Y. H. Chang, "Helicopter $H_{\infty}$ control design with robust flying quality," Aerospace Science and Technology, vol. 7, no. 2, pp. 159-169, 2003.

[11] A. Isidori, L. Marconi, and A. Serrani, "Robust nonlinear motion control of a helicopter," IEEE Transactions on Automatic Control, vol. 48, no. 3, pp. 413-426, 2003.

[12] A. Budiyono and S. S. Wibowo, "Optimal tracking controller design for a small scale helicopter," Journal of Bionic Engineering, vol. 4, no. 4, pp. 271-280, 2007.

[13] S. S. Ge, B. Ren, K. P. Tee, and T. H. Lee, "Approximation-based control of uncertain helicopter dynamics," IET Control Theory \& Applications, vol. 3, no. 7, pp. 941-956, 2009.

[14] W. Adiprawita, A. S. Ahmad, and J. Sembiring, "Automated flight test and system identification for rotary wing aerial platform using frequency responses analysis," Journal of Bionic Engineering, vol. 4, no. 4, pp. 237-244, 2007.

[15] L. David, R. Gerardo, S. Anand, L. Rogelio, and G. Alfredo, "Robustness margin for attitude control of a four rotor minirotorcraft: case of study," Mechatronics, vol. 20, no. 1, pp. 143-152, 2010.

[16] P. Apkarian, C. Champetier, and J.-F. Magni, "Design of a helicopter output feedback control law using modal and structuredrobustness techniques," International Journal of Control, vol. 50, no. 4, pp. 1195-1215, 1989. 
[17] D. Martens, "Neural networks as a tool for the assessment of human pilot behaviour in wind shear," Aerospace Science and Technology, vol. 3, no. 1, pp. 39-48, 1999.

[18] A. Douik, "Optimized eigenstructure assignment by ant system and LQR approaches," Computer Science and Applications, vol. 5, no. 4, pp. 45-56, 2008.

[19] S. Suzuki, K. Hayashi, and T. Uemura, "Analysis of human pilot behavior at landing with neural network combined with GA," Japan Society For Aeronautical and Space Sciences, vol. 51, pp. 109-113, 2003.

[20] X. S. Lei and J. J. Li, "An adaptive altitude information fusion method for autonomous landing processes of small unmanned aerial rotorcraft," Sensors, vol. 12, no. 10, pp. 13212-13214, 2012.

[21] X. S. Lei and Y. H. Du, "A linear domain system identification for small unmanned aerial rotorcraft based on adaptive genetic algorithm," Journal of Bionic Engineering, vol. 7, no. 2, pp. 142$149,2010$. 


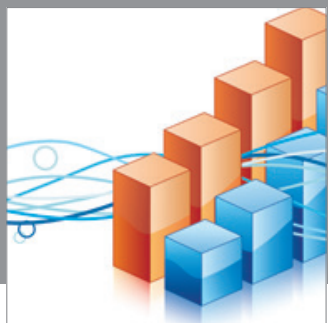

Advances in

Operations Research

mansans

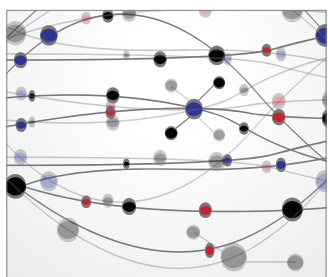

The Scientific World Journal
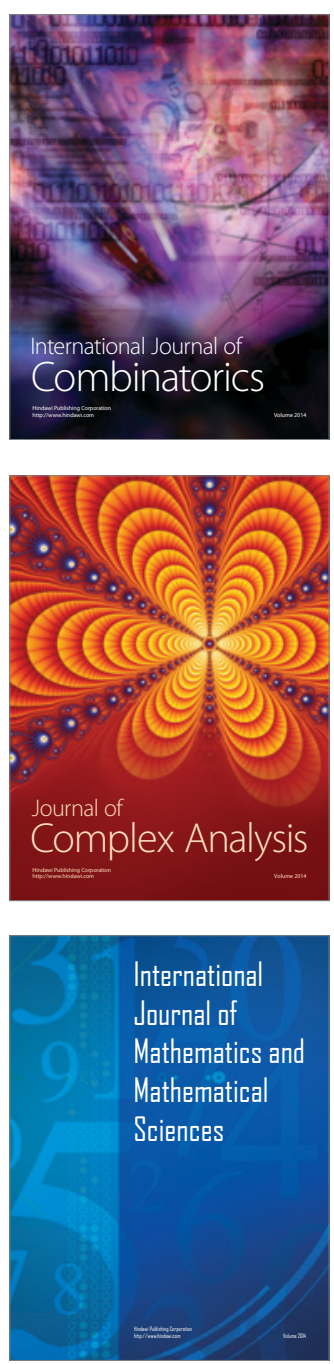
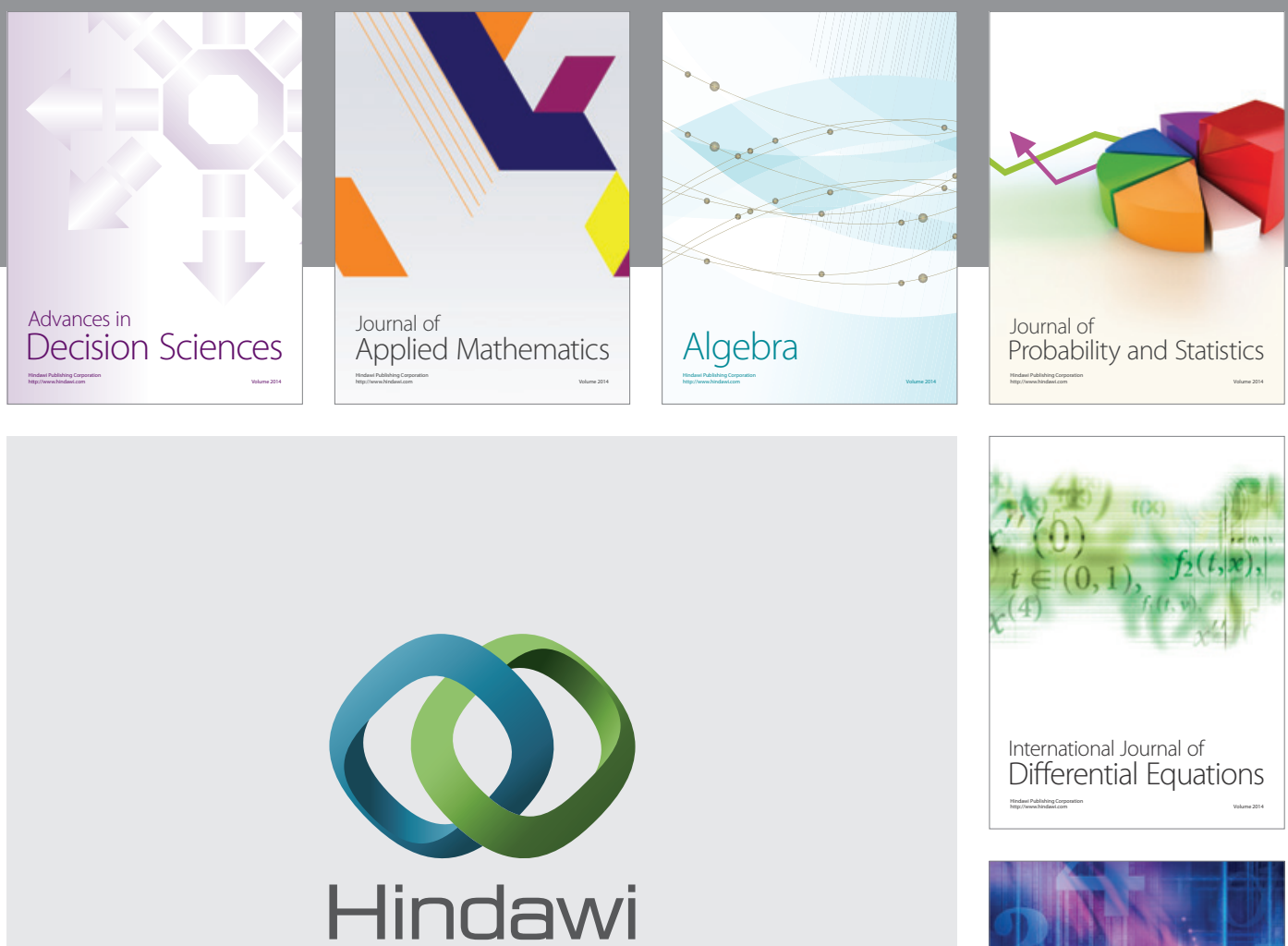

Submit your manuscripts at http://www.hindawi.com
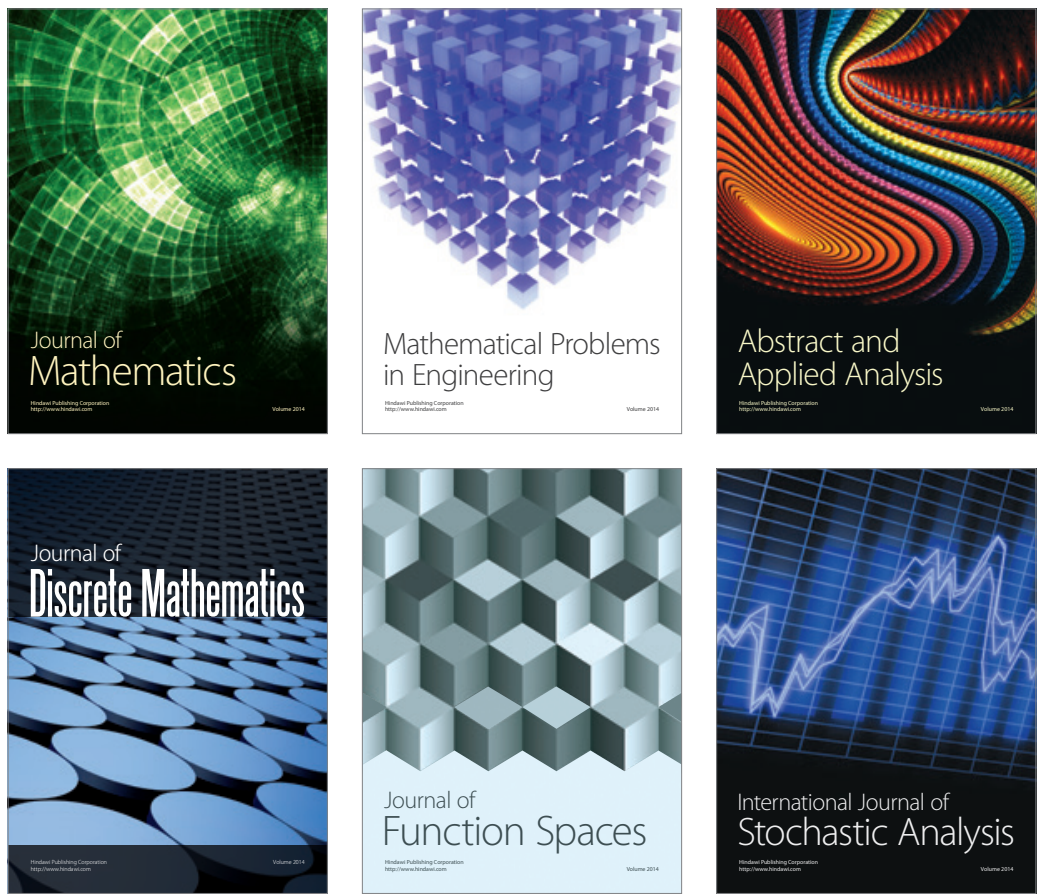

Journal of

Function Spaces

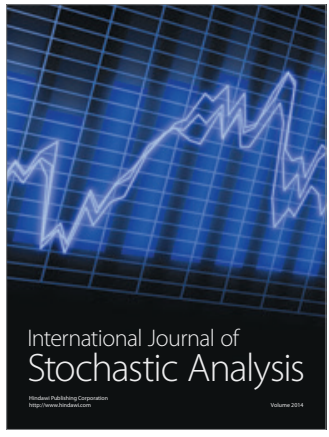

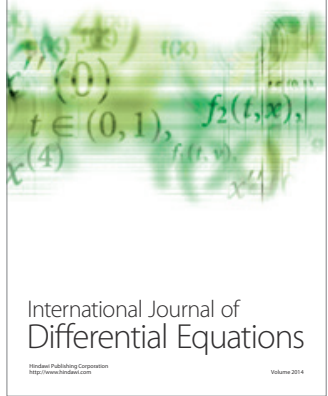
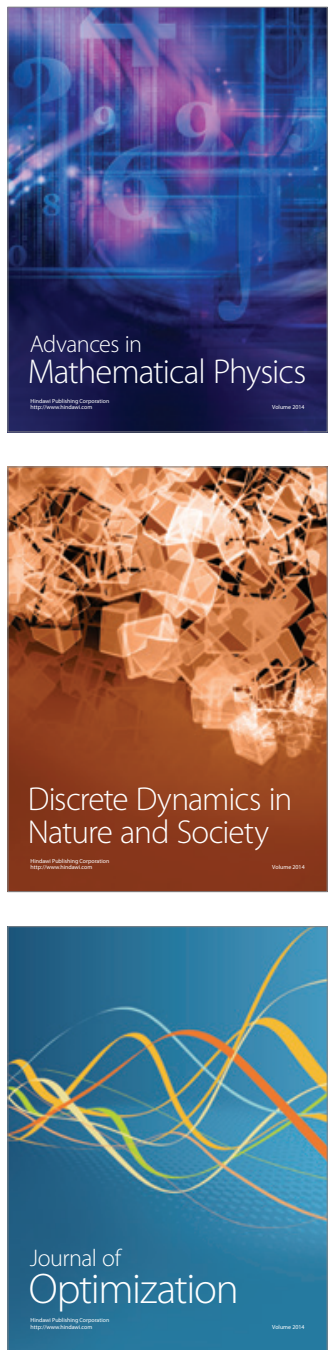